\title{
Estado da arte em ambientes u-learning cientes de contexto: uma Revisão Sistemática da Literatura
}

\author{
Roger S. Machado ${ }^{1}$, Felipe L. da Rosa ${ }^{1}$, Tiago T. Primo ${ }^{1}$, \\ Ana Marilza Pernas ${ }^{1}$, Adenauer C. Yamin ${ }^{1}$ \\ ${ }^{1}$ Universidade Federal de Pelotas (UFPel) \\ \{rdsmachado, fldrosa,tiago.primo, adenauer, marilza\}@inf.ufpel.edu.br
}

\begin{abstract}
The purpose of u-learning environments is to continuously process the student's actions troght lifelong experiences, allowing the learning to accompany and adapt the student's needs during life. Motivated by this scenario, this paper aims to present the state of the art in context-aware u-learning environments. For this, we explored a Systematic Literature Review. This review analyzes works published between 2012 and 2017. During the review process, we identified 217 papers and selected five papers that proposes context-aware $u$ learning environments. The selected papers are presented, discussed and analyzed.
\end{abstract}

Resumo. Ambientes u-learning tem como proposta o processamento contínuo das experiências de aprendizado, permitindo com que este se adapte as necessidades do estudante ao longo de sua vida. Motivado por este cenário, esse artigo tem o objetivo de apresentar o estado da arte em ambientes $u$-learning cientes de contexto. Para isso, é explorada uma Revisão Sistemática da Literatura. Nessa revisão são analisados trabalhos publicados entre 2012 e 2017. Durante o processo de revisão foram identificados 217 artigos, sendo selecionados ao final cinco trabalhos que propõem ambientes u-learning cientes de contexto. Os trabalhos selecionados são apresentados, discutidos e analisados.

\section{Introdução}

Com o crescimento da quantidade de dispositivos conectados e informação disponibilizada, a questão de como tornar os processos de aprendizagem mais eficientes e convenientes baseados no processamento destas informações tornou-se fundamental [Sánchez Guinea et al. 2016]. Cenários como este dão vida ao aprendizado ubíquo ( $u$ learning - ubiquitous learning) como proposto por [Hwang 2014]. Nos ambientes $u$ learning, o contexto dos usuários é monitorado constantemente, e utilizado pelo sistema para adaptar as atividades propostas de modo a beneficiar o aluno e seu acesso a conteúdos personalizados as suas necessidades educacionais [Souabni et al. 2016].

Ambientes $u$-learning trazem possibilidades de inferência e compreensão sobre o aprendizado dos alunos. Principalmente devido ao uso de tecnologias móveis em praticamente todas as suas atividades diárias. Fato este que torna imperativo que tais tecnologias possam ser exploradas para trazer ganhos práticos ao aprendizado [Chiu et al. 2017]. Por outro lado, este cenário traz diversos desafios tecnológicos, como a complexidade em se adquirir, processar e reagir de forma eficiente as informações que mudam a todo o momento, e que possuem uma escala crescente de número de usuários [Selviandro et al. 2016]. 
VII Congresso Brasileiro de Informática na Educação (CBIE 2018)

Anais do XXIX Simpósio Brasileiro de Informática na Educação (SBIE 2018)

O desafio de um ambiente de u-learning não é somente fornecer informações ou serviços de aprendizagem a qualquer hora e em qualquer lugar, mas também levar as informações certas no momento certo, e para a pessoa certa [Fischer 2012]. Assim, o ambiente de u-learning deve considerar o perfil de cada aluno, adaptando informações e serviços para corresponder às necessidades específicas de cada um [Adomavicius and Tuzhilin 2005].

Desta forma, a ciência de contexto torna-se uma característica fundamental para os ambientes u-learning [Huang et al. 2011]. Onde a ciência de contexto é a capacidade de utilizar informações contextuais para prover informações e serviços relevantes para o usuário, onde relevância depende da tarefa que o usuário está executando [Alegre et al. 2016].

Diversos são os benefícios da utilização de ambientes $u$-learning cientes de contexto para ajudar os alunos a melhorar sua capacidade de resolver problemas no mundo real [Bedi et al. 2010]. O objetivo deste artigo é apresentar o estado da arte na área de ambientes u-learning cientes de contexto apontando para desafios e oportunidades de pesquisa na área.

Para alcançar esse objetivo, é realizada uma Revisão Sistemática da Literatura (RSL), cujos procedimentos necessários para sua consecução são discutidos, facultando sua reconstrução com outros critérios. Como produto, foram identificados trabalhos representativos na área no período entre 2012 e 2017, sendo registrados os retornos obtidos em três diferentes bases de indexação. Ao final da RSL, foram selecionados cinco trabalhos que propõem ambientes $u$-learning cientes de contexto. Os trabalhos selecionados são apresentados, discutidos e analisados.

Este artigo está dividido da seguinte forma: a Seção 2 discute a RSL realizada; a Seção 3 apresenta os trabalhos selecionados durante a RSL, onde são analisadas as principais características de cada trabalho; a Seção 4 discute as principais características observadas durante o desenvolvimento dessa RSL; e, por fim, a Seção 5 apresenta as considerações finais e os trabalhos futuros.

\section{Estado da arte em ambientes u-learning}

A Revisão Sistemática da Literatura (RSL) é uma metodologia de pesquisa que prevê a execução de uma série de procedimentos para identificar um conjunto de artigos que traz uma prospecção criteriosa do tema de pesquisa abordado [Kitchenham and Charters 2007]. Uma característica desta metodologia de pesquisa é que todos os seus procedimentos são registrados, permitindo que o estudo realizado seja reproduzível por outros pesquisadores.

A RSL desenvolvida neste trabalho foi realizada com base nos conceitos originais encontrados em [Biolchini et al. 2005] e [Kitchenham and Charters 2007]. Para auxiliar no desenvolvimento da RSL foi utilizada a ferramenta StArt ${ }^{1}$, desenvolvida pela Universidade Federal de São Carlos (UFSCAR).

O principal objetivo da RSL realizada é identificar o estado da arte em ambientes u-learning cientes de contexto.

\footnotetext{
${ }^{1}<$ http://lapes.dc.ufscar.br/tools/start_tool>
} 
VII Congresso Brasileiro de Informática na Educação (CBIE 2018)

Anais do XXIX Simpósio Brasileiro de Informática na Educação (SBIE 2018)

\subsection{Bases digitais e string de busca}

As bases digitais escolhidas para a busca dos artigos nesta RSL foram: IEEE Xplore ${ }^{2}$, Web of Science ${ }^{3}$ e ACM Digital Library ${ }^{4}$. A escolha das bases de indexação se deu devido a sua importância, bem como por cobrirem uma ampla parte dos periódicos e artigos de conferências publicados na área de ambientes $u$-learning cientes de contexto.

Para a pesquisa, foi utilizada uma string de busca que combina as principais palavras chaves consideradas pertinentes a RSL. A mesma string foi utilizada nas três bases digitais, e os mecanismos de busca foram configurados para buscar pela string no título, abstract e palavras-chave dos artigos. A string de busca utilizada foi:

\section{((“context aware") OR (“context awareness")) AND ("learning") AND ("environment") AND ((“u-learning") OR ("ubiquitous learning”))}

\subsection{Processo de análise}

O processo de análise dos artigos retornados pelas bases digitais é apresentado na Figura 1 , onde pode-se visualizar suas cinco etapas.

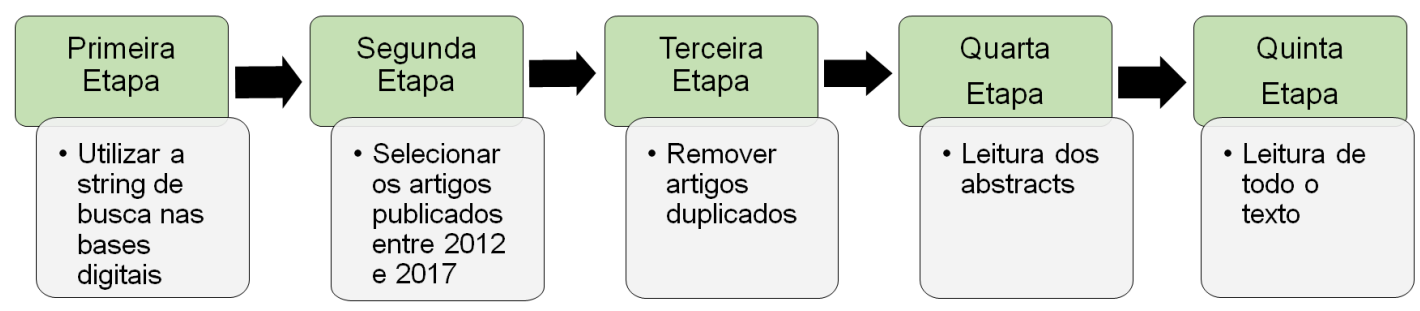

Figura 1. Processo de análise da RSL realizada.

Na primeira etapa foram realizadas buscas sem a aplicação de nenhum filtro, onde somente foi utilizada a string de busca em cada base digital. Já na segunda etapa optou-se por restringir o escopo da busca, analisando somente publicações realizadas entre os anos de 2012 e 2017, um período de tempo considerado ainda atual e relevante para publicações da área.

A terceira etapa da análise das publicações consistiu da detecção dos artigos duplicados. A ferramenta StArt, utilizada no trabalho, classifica automaticamente artigos com o mesmo título e mesmos autores como duplicados. Porém, em alguns casos o título do artigo ou os nomes dos autores podem estar escritos de maneira diferente nas bases digitais, fazendo com que a ferramenta não consiga realizar a detecção automática. Para resolver esta questão, foi realizado o processo de verificação manual dos artigos duplicados, assim, o título e o nome dos autores de cada artigo encontrado foram lidos e, dependendo do caso, o artigo foi classificado como duplicado.

$\mathrm{Na}$ quarta etapa da análise foi feita a leitura do abstract de cada publicação encontrada. Enquanto que na quinta e última etapa foram lidos os artigos na íntegra.

\footnotetext{
${ }^{2}<$ http: // ieeexplore.ieee.org $>$

${ }^{3}<$ https: / / webofknowledge. com>

${ }^{4}$ <https: / / dl .acm.org>
} 
VII Congresso Brasileiro de Informática na Educação (CBIE 2018)

Anais do XXIX Simpósio Brasileiro de Informática na Educação (SBIE 2018)

\subsection{Critérios de inclusão e exclusão}

Diversos critérios de inclusão e exclusão de artigos foram utilizados nesta RSL. Os critérios de inclusão foram:

- ser um artigo publicado entre 2012 e 2017;

- propor um ambiente de u-learning ciente de contexto;

- apresentar aplicação prática do ambiente proposto.

Por sua vez, os critérios de exclusão considerados durante a revisão foram:

- artigo publicado antes de 2012;

- o artigo não tem como foco a u-learning;

- o artigo propõe uma aplicação muito específica;

- o artigo não apresenta validação do ambiente proposto;

- o artigo possui menos de seis páginas;

- o artigo registra pequenas diferenças de outro artigo.

O último critério de exclusão foi utilizado para excluir os trabalhos preliminares de uma mesma pesquisa, sendo selecionado somente o trabalho mais atual. Para aplicação desse critério foi analisado todo o texto dos artigos, verificando se os artigos tratavam da mesma pesquisa, registrando apenas pequenas diferenças entre eles.

\subsection{Visão geral dos dados obtidos na RSL}

A Tabela 1 apresenta um resumo quantitativo da revisão sistemática, com o número de artigos tratados em cada etapa desta RSL.

Tabela 1. Artigos retornados por cada etapa da RSL.

\begin{tabular}{c|c|c|c|c}
\hline Primeira Etapa & Segunda Etapa & Terceira Etapa & Quarta Etapa & Quinta Etapa \\
\hline 217 & 98 & 64 & 31 & 5 \\
\hline
\end{tabular}

A Tabela 2 mostra uma comparação quantitativa do resultado das pesquisas nas três bases digitais, apresentando os números de: (i) artigos retornados sem aplicação de filtro; (ii) artigos publicados entre os anos de 2012 e 2017; (iii) artigos rejeitados; (iv) artigos aceitos. Destaca-se que os artigos duplicados estão contabilizados na tabela 2.

Tabela 2. Comparação quantitativa das publicações nas três bases digitais.

\begin{tabular}{c|c|c|c|c}
\hline Base Digital & Total & Com filtro de data & Rejeitados & Aceitos \\
\hline IEEE & 63 & 26 & 24 & 3 \\
\hline Web of Science & 92 & 46 & 41 & 5 \\
\hline ACM & 62 & 26 & 24 & 2 \\
\hline
\end{tabular}

A Tabela 3 apresenta uma visão geral do número de artigos que foram excluídos com base em cada critério de exclusão. Conforme pode ser visualizado, o critério que excluiu o maior número de trabalhos foi o de eliminar artigos que não foram publicados entre 2012 e 2017, sendo excluídos 107 artigos. 
VII Congresso Brasileiro de Informática na Educação (CBIE 2018)

Anais do XXIX Simpósio Brasileiro de Informática na Educação (SBIE 2018)

Tabela 3. Número de artigos excluídos por cada critério.

\begin{tabular}{c|c}
\hline Critério de exclusão & Numero de Artigos \\
\hline artigo publicado antes de 2012 & 107 \\
\hline o artigo não tem como foco a u-learning & 20 \\
\hline o artigo propõe uma aplicação muito específica & 18 \\
\hline o artigo não apresenta validação do ambiente proposto & 12 \\
\hline o artigo possui menos de seis páginas & 7 \\
\hline o artigo registra pequenas diferenças de outro artigo & 3 \\
\hline
\end{tabular}

Ao final da RSL realizada, cinco artigos foram selecionados. A Tabela 4 mostra as principais informações sobre os artigos selecionados, sendo apresentados: (i) autores; (ii) título; (iii) ano de publicação; (iv) conferência ou periódico que foi publicado; e (v) base digital que retornou o artigo.

Tabela 4. Artigos selecionados na RSL.

\begin{tabular}{|c|c|c|c|c|}
\hline Autores & Título & Ano & Conferência /Periódico & Base Digital \\
\hline $\begin{array}{c}\text { [Hijazi and } \\
\text { Itmazi 2013] }\end{array}$ & $\begin{array}{l}\text { Crawler based context aware } \\
\text { model for distributed } \\
\text { e-courses through ubiquitous } \\
\text { computing at higher education } \\
\text { institutes }\end{array}$ & 2013 & $\begin{array}{c}\text { Fourth International } \\
\text { Conference on } \\
\text { e-Learning }\end{array}$ & $\begin{array}{l}\text { IEEE e Web } \\
\text { of Science }\end{array}$ \\
\hline $\begin{array}{l}\text { [Wagner et } \\
\text { al. 2014] }\end{array}$ & $\begin{array}{l}\text { A model for profile } \\
\text { management applied to } \\
\text { ubiquitous learning } \\
\text { environments }\end{array}$ & 2014 & $\begin{array}{l}\text { Expert Systems with } \\
\text { Applications }\end{array}$ & $\begin{array}{l}\mathrm{ACM} \text { e Web } \\
\text { of Science }\end{array}$ \\
\hline $\begin{array}{l}\text { [Atif et al. } \\
\text { 2015] }\end{array}$ & $\begin{array}{l}\text { Building a smart campus to } \\
\text { support ubiquitous learning }\end{array}$ & 2015 & $\begin{array}{c}\text { Journal of Ambient } \\
\text { Intelligence and } \\
\text { Humanized Computing }\end{array}$ & $\begin{array}{l}\text { Web of } \\
\text { Science }\end{array}$ \\
\hline $\begin{array}{c}\text { [Pernas et al. } \\
\text { 2015] }\end{array}$ & $\begin{array}{l}\text { Integrated Infrastructure for } \\
\text { Ubiquitous Learning }\end{array}$ & 2015 & $\begin{array}{c}\text { IEEE Revista } \\
\text { Iberoamericana de } \\
\text { Tecnologias del } \\
\text { Aprendizaje }\end{array}$ & $\begin{array}{l}\text { IEEE e Web } \\
\text { of Science }\end{array}$ \\
\hline $\begin{array}{l}\text { [Hsu et al. } \\
\text { 2016] }\end{array}$ & $\begin{array}{l}\text { Development and evaluation } \\
\text { of an active learning support } \\
\text { system for context-aware } \\
\text { ubiquitous learning }\end{array}$ & 2016 & $\begin{array}{l}\text { IEEE Transactions on } \\
\text { Learning Technologies }\end{array}$ & $\begin{array}{l}\text { ACM, IEEE } \\
\text { e Web of } \\
\text { Science }\end{array}$ \\
\hline
\end{tabular}

\section{Artigos selecionados}

Após a realização da RSL apresentada anteriormente, foram selecionados cinco artigos. Os trabalhos selecionados são apresentados a seguir, sendo exploradas as suas principais funcionalidades. 
VII Congresso Brasileiro de Informática na Educação (CBIE 2018)

Anais do XXIX Simpósio Brasileiro de Informática na Educação (SBIE 2018)

\subsection{Crawler ciente de contexto para recuperar materiais de ensino de bases abertas}

Em [Hijazi and Itmazi 2013] é proposto um ambiente de $u$-learning ciente de contexto, que serviria como uma atualização do sistema de e-learning (electronic learning) já existente. Como diferencial, o artigo propõe o desenvolvimento de um crawler focado em recuperar materiais de ensino de bases abertas.

O modelo baseia-se no uso dos recursos disponíveis para fornecer o material do e-curso, atualizar o servidor de e-learning com o contexto dos alunos e atualizar o repositório de e-learning usando o crawler desenvolvido.

Com o uso do crawler, o ambiente de $u$-learning estaria constantemente atualizado com novos materiais. O crawler é responsável por rastrear os sites acadêmicos (sites de recursos educacionais abertos), fazer o download dos recursos educacionais relacionados (por exemplo, documentos de artigos) e indexá-los com base nas palavras-chave encontradas.

Os resultados da pesquisa mostraram que o uso de diferentes recursos de entrega de conteúdo ajuda os alunos a obterem materiais mais adequados, e assim, podendo oferecer e-cursos de melhor qualidade.

\subsection{Modelo de gerenciamento de perfil aplicado a ambientes u-learning}

O trabalho de [Wagner et al. 2014] apresenta a concepção e desenvolvimento do eProfile, um modelo para prover gerenciamento de perfis de usuários em ambientes interoperáveis baseado no monitoramento de tais usuários. O termo "monitoramento" neste caso se refere a informações contextuais históricas dos usuários, que são armazenadas e avaliadas pelo sistema. As contribuições desse modelo são o uso de trilhas para extração de perfis e a capacidade de gerenciar regras de inferência para geração de perfis.

O eProfile é composto por quatro agentes autônomos (Communicator, Configurator, Converter e Reasoner). Os autores destacam que o uso de agentes simplificou o processamento dos dados instanciados em uma ontologia de domínio e a execução das regras de inferências.

O agente Communicator é responsável por compartilhar o perfil de entidade com os aplicativos. Já o Configurator recebe as entradas dos aplicativos, por exemplo, modelo de entidade e regras. Enquanto o Converter é responsável por receber dados no formato do gerenciador de trilhas e convertê-los para o formato da ontologia eOntoTrail.

Para inferência das trilhas é utilizado o Reasoner, o qual gera um perfil de entidade que consiste em uma extensão de uma ontologia de perfil denominada eOnto-Profile. Essa ontologia utiliza as informações de perfil inferidas da trilha, de acordo com o modelo de entidade definido pela aplicação.

Os autores utilizaram o eProfile em conjunto com um ambiente de u-learning, onde o perfil do aluno é provido pelo seu eProfile e sugestões de cursos de disciplinas são feitos baseados neste perfil, que é atualizado constantemente baseado no rastro do aluno.

\subsection{Ambiente u-learning utilizando realidade aumentada}

Em [Atif et al. 2015], é apresentada uma proposta de desenvolvimento de um campus inteligente, que utiliza ciência de contexto e tecnologias de realidade aumentada e comunicação por campo de proximidade para prover um ambiente de $u$-learning. 
VII Congresso Brasileiro de Informática na Educação (CBIE 2018)

Anais do XXIX Simpósio Brasileiro de Informática na Educação (SBIE 2018)

É proposta uma arquitetura que define vários componentes e suas interoperações em um ambiente educacional inteligente. A abordagem do campus inteligente é apresentada como uma composição de espaços de aprendizagem ambiental, que são ambientes onde os recursos de aprendizagem física são aumentados com serviços digitais e sociais. Um modelo desses espaços é apresentado, o qual pode aproveitar futuros ambientes $u$ learning. Uma das características distintivas desse modelo é a capacidade de liberar o valor instrucional das estruturas físicas circundantes.

No artigo ainda é proposto uma expansão da descrição para fornecer objetos PLOM (Pervasive LOM) cuja capacidade de representação é esticada para entidades de educação física, como uma sala de aula inteligente, pôsteres ou laboratório, ou até mesmo livros de realidade aumentada.

$\mathrm{O}$ ambiente de $u$-learning foi avaliado com o intuito de analisar o desempenho dos blocos de construção no modelo de campus inteligente proposto. Os resultados da avaliação foram considerados como promissores pelos autores, indicando que sua abordagem é válida.

\subsection{Infraestrutura integrando um ambiente e-learning e um middleware ubíquo}

O artigo de [Pernas et al. 2015] apresenta uma arquitetura para $u$-learning, a qual emprega o paradigma de acesso a dados baseado em ontologias (OBDA) para resolver problemas relacionados à integração de dados e acesso a fontes de dados em estruturas complexas. As técnicas de OBDA são exploradas para permitir o acesso e o mapeamento de dados contextuais disponíveis em um ambiente de e-learning e sua integração com um middleware para computação ubíqua, assim proporcionando um ambiente de $u$-learning.

Para realizar a integração é utilizado um modelo conceitual, o qual consiste de uma rede de ontologias relacionadas entre si através de meta-relacionamentos. A rede de ontologias utilizadas visa apoiar uma estratégia adaptativa baseada na detecção automática da situação do aluno, modelando os elementos contextuais de três áreas distintas: (i) o domínio do aluno, que representa o modelo do aluno e sua situação de aprendizagem; (ii) domínio educacional, que representa o modelo de domínio; e (iii) domínio tecnológico, que modela o ambiente físico e tecnológico.

A aplicabilidade da proposta foi avaliada com o uso de um estudo de caso, onde foi descrito que o aluno passaria a utilizar o ambiente de e-learning sem nenhuma ação extra necessária. Enquanto o middleware para computação ubíqua passaria a gerenciar o acesso de vários alunos e processar o contexto de cada um separadamente, como aplicações ubíquas distintas. No cenário apresentado é detectado por meio de regras SWRL (Semantic Web Rule Language) uma situação de aprendizado que precisa de uma intervenção com a ação de recomendar um material extra baseado no estilo de aprendizagem do aluno.

\subsection{Sistema ativo de apoio à aprendizagem ciente de contexto}

Em [Hsu et al. 2016] é apresentada uma proposta de desenvolvimento de um ambiente de $u$-learning ciente de contexto denominado ALESS, que funcionaria como um sistema adaptativo de suporte a navegação. O autor sustenta a teoria de que a maioria dos sistemas de u-learning funcionam de modo "passivo", ou seja, o usuário precisa se locomover até certas tags para iniciar o aprendizado. 
VII Congresso Brasileiro de Informática na Educação (CBIE 2018)

Anais do XXIX Simpósio Brasileiro de Informática na Educação (SBIE 2018)

Em sua proposta, o aprendizado seria "ativo", ou seja, não seria necessária a utilização de tags ou $Q R$ codes. O ALESS consiste em duas partes, o servidor e o cliente. O servidor responde para registrar o contexto do ambiente e determinar os caminhos de aprendizado. O cliente responde para enviar as informações de localização e lidar com as solicitações dos alunos e do servidor.

Uma grande quantidade de informações de contexto é usada em ALESS, incluindo a localização, a capacidade atual do objeto de aprendizado, o tempo disponível, etc. A ALESS é capaz de fornecer ativamente o suporte de aprendizagem necessário para alunos individuais quando eles abordarem as metas de aprendizado do mundo real correspondentes.

Como caso de uso, foi utilizado uma visita de alunos do ensino médio a um museu onde, ao invés de escanear os códigos presentes fisicamente no museu (que são disponibilizados pela gerência do museu), os alunos podem utilizar o ALESS para se deslocar em uma versão virtual do museu, aprendendo a todo o momento e eliminando problemas como superlotação de uma obra específica. Os resultados experimentais mostraram que, com a ajuda da ALESS, os alunos aprenderam com mais eficiência e alcançaram melhor desempenho de aprendizado.

Como conclusão, foi constatado que embora o sistema tenha obtido resultados similares a outros sistemas em quesito de aprendizado, o tempo de ócio do aluno diminuiu, já que o mesmo não precisa gastar tempo se deslocando de uma tag a outra.

\section{Discussão}

Durante a RSL realizada observou-se que alguns trabalhos apesar de conterem os termos da string de pesquisa, eles não tinham como foco a u-learning. Desta forma, foi aplicado um filtro para eliminar esses trabalhos, sendo eliminados ao total 20 artigos.

Outra característica interessante notada é que muitos trabalhos propõem ambientes $u$-learning muito específicos, por exemplo, para estudar as obras de um determinado museu, ou então para uma turma específica estudar somente um conteúdo sobre plantas. Devido ao interesse de buscar ambientes u-learning mais genéricos foi aplicado um filtro que remove trabalhos que propõem aplicações muito específicas, sendo removidos 18 artigos.

Destaca-se ainda que foi observado que alguns artigos possuem propostas de ambientes $u$-learning que não apresentam nenhum tipo de validação da proposta. Além disso, alguns artigos não mostram nem mesmo um tipo de aplicação que poderia ser utilizada no ambiente proposto. Desta forma, foi aplicado um filtro para remover estes trabalhos, onde foram removidos 12 artigos.

Como pode ser observado nesta RSL, diferentes trabalhos propõem ambientes u-learning, e embora todos sigam a premissa de desenvolver um sistema que facilite o aprendizado ubíquo, cada um se utiliza de uma abordagem diferente. Ainda assim, existem diversas similaridades entre os trabalhos analisados. Como exemplo pode-se destacar o fato de [Wagner et al. 2014], [Pernas et al. 2015] e [Atif et al. 2015] utilizarem ontologias como forma de representação e processamento dos dados contextuais, enquanto [Hijazi and Itmazi 2013] e [Hsu et al. 2016] não especificam a estratégia de representação dos dados, falando apenas que são utilizados repositórios para armazenamento. 
VII Congresso Brasileiro de Informática na Educação (CBIE 2018)

Anais do XXIX Simpósio Brasileiro de Informática na Educação (SBIE 2018)

Além disso, tanto [Wagner et al. 2014] quanto [Atif et al. 2015] propõe a criação de um ambiente virtual de aprendizado, sistema que os alunos utilizariam para seu aprendizado. Em contrapartida, [Pernas et al. 2015] apresenta uma proposta de integrar dois componentes já em uso, um ambiente de e-learning e um middleware para computação ubíqua, com o intuito de fornecer um ambiente de u-learning. Já [Hijazi and Itmazi 2013] e [Hsu et al. 2016] focam na criação de uma arquitetura de $u$-learning, que será utilizada junto com tags e instrutores do mundo real.

Embora existam várias propostas de ambientes de u-learning, nota-se que os esforços de pesquisa relacionados ao tema ainda são necessários, principalmente com foco no desenvolvimento de abordagens que sejam flexíveis. Uma vez que as propostas atuais, muitas vezes, tratam de questões específicas, não possuindo por exemplo, vocabulários comuns para representação dos dados presentes em ambientes educacionais e mecanismos que possibilitem a coleta de dados de diferentes tipos de aplicações.

\section{Considerações finais}

Este artigo apresentou o estado da arte, sistematizando tendências de pesquisa em ambientes u-learning cientes de contexto. Para tanto, foi explorada uma Revisão Sistemática da Literatura (RSL), contribuindo tanto pela identificação de trabalhos relevantes na área bem como pela especificação de critérios para sua seleção. Nesta RSL foram analisados trabalhos publicados nos últimos seis anos, analisando os trabalhos entre 2012 e 2017.

Durante a RSL foram identificados 217 trabalhos, sendo selecionados ao final cinco trabalhos que propõem ambientes u-learning cientes de contexto. Os trabalhos selecionados foram apresentados e discutidos, sendo realizada uma análise comparativa entre as características de cada trabalho.

Como trabalho futuro destaca-se a capacitação de uma plataforma virtual de aprendizagem em um ambiente de u-learning. Para isso será desenvolvido um vocabulário semântico, para representação dos dados presentes na plataforma, e um mecanismo de coleta de dados, realizando o monitoramento e o processamento dos mesmos, e assim obtendo a ciência de contexto. Com essa obtenção serão oferecidos serviços de recomendação para o estudante com base nos contextos disponíveis, disponibilizando funcionalidades que facilitem o aprendizado do aluno.

\section{Agradecimentos}

O presente trabalho foi realizado com apoio da CAPES (Programa Nacional de Cooperação Acadêmica - Procad) e da FAPERGS (Programa Pesquisador Gaúcho - PqG). Roger S. Machado é Bolsista FAPERGS/CAPES - BRASIL, nível doutorado.

\section{Referências}

Adomavicius, G. and Tuzhilin, A. (2005). Personalization technologies: A processoriented perspective. Communications of the ACM, 48(10):83-90.

Alegre, U., Augusto, J. C., and Clark, T. (2016). Engineering context-aware systems and applications: A survey. Journal of Systems and Software, 117:55-83.

Atif, Y., Mathew, S. S., and Lakas, A. (2015). Building a smart campus to support ubiquitous learning. Journal of Ambient Intelligence and Humanized Computing, 6(2):223238. 
VII Congresso Brasileiro de Informática na Educação (CBIE 2018)

Anais do XXIX Simpósio Brasileiro de Informática na Educação (SBIE 2018)

Bedi, P., Banati, H., and Thukral, A. (2010). Social semantic retrieval and ranking of eresources. In 2010 International Conference on Advances in Recent Technologies in Communication and Computing, pages 343-347.

Biolchini, J., Mian, P. G., Natali, A. C. C., and Travassos, G. H. (2005). Systematic review in software engineering. System Engineering and Computer Science Department COPPE/UFRJ, Technical Report ES, 679(05):45.

Chiu, C.-K., Tseng, J. C., and Hsu, T.-Y. (2017). Blended context-aware ubiquitous learning in museums: Environment, navigation support and system development. Personal Ubiquitous Comput., 21(2):355-369.

Fischer, G. (2012). Context-aware systems: The "right" information, at the "right" time, in the "right" place, in the "right" way, to the "right" person. In Proceedings of the International Working Conference on Advanced Visual Interfaces, AVI '12, pages 287294, Capri Island, Italy. ACM.

Hijazi, H. W. and Itmazi, J. A. (2013). Crawler based context aware model for distributed e-courses through ubiquitous computing at higher education institutes. In Fourth International Conference on e-Learning "Best Practices in Management, Design and Development of e-Courses: Standards of Excellence and Creativity”, pages 9-14. IEEE.

Hsu, T.-Y., Chiou, C.-K., Tseng, J. C., and Hwang, G.-J. (2016). Development and evaluation of an active learning support system for context-aware ubiquitous learning. IEEE Transactions on Learning Technologies, 9(1):37-45.

Huang, Y.-M., Chiu, P.-S., Liu, T.-C., and Chen, T.-S. (2011). The design and implementation of a meaningful learning-based evaluation method for ubiquitous learning. Computers \& Education, 57(4):2291-2302.

Hwang, G.-J. (2014). Definition, framework and research issues of smart learning environments-a context-aware ubiquitous learning perspective. Smart Learning Environments, 1(1):4.

Kitchenham, B. and Charters, S. (2007). Guidelines for performing systematic literature reviews in software engineering. Joint report, Keele University and Durham University.

Pernas, A. M., Gasparini, I., Pires, L. V., Lopes, J. L. B., Yamin, A. C., and de Oliveira, J. P. M. (2015). Integrated infrastructure for ubiquitous learning. IEEE Revista Iberoamericana de Tecnologias del Aprendizaje, 10(3):92-99.

Sánchez Guinea, A., Nain, G., and Le Traon, Y. (2016). A systematic review on the engineering of software for ubiquitous systems. J. Syst. Softw., 118(C):251-276.

Selviandro, N., Sabariah, M. K., and Saputra, S. (2016). Context awareness system on ubiquitous learning with case based reasoning and nearest neighbor algorithm. In 4th International Conference on Information and Communication Technology.

Souabni, R., Saadi, I. B., Salah, N. B., Ghezala, H. B., et al. (2016). On the evaluation of quality of situation (qosi) in situation-aware ubiquitous learning environment. In Advanced Information Networking and Applications (AINA), pages 239-246. IEEE.

Wagner, A., Barbosa, J. L. V., and Barbosa, D. N. F. (2014). A model for profile management applied to ubiquitous learning environments. Expert Systems with Applications, 41(4):2023-2034. 